\title{
Probing rotation in pulsating stars from space
}

\author{
Jaymie M. Matthews \\ Department of Physics \& Astronomy, University of British Columbia, \\ 6224 Agricultural Road, Vancouver V6T 1Z1 Canada
}

\begin{abstract}
At the time of the last Stellar Rotation symposium in 1969, the map of known pulsators in the H-R Diagram covered a lot less territory, being confined mainly to the classical instability strip. Among the new classes of pulsators are the strongly magnetic roAp (rapidly oscillating Ap) stars whose pulsation amplitudes and phases are modulated with their rotation periods. Among the classical pulsators, the $\delta$ Scuti stars are now known to show many more eigenmodes and frequency splitting than was ever recognised three decades ago. Both are examples of the diagnostic potential and severe challenges to detect and interpret correctly rotational fine structure in the pulsational frequency spectrum. In a pulsating star, rotation can perturb the structure or dynamics, and it can split degeneracies to serve as a diagnostic of pulsational modes. One tool which should help us exploit the potential and overcome the challenges of studying the interaction between rotation and pulsation is Canada's MOST (Microvariability \& Oscillations of STars) microsatellite. MOST is a small optical telescope and ultraprecise CCD photometer designed to detect and characterise acoustic $(p$-mode) oscillations with periods of minutes and amplitudes as low as 1 micromagnitude in bright stars. While customised to conduct asteroseismology of solar-type stars, the MOST mission will also include roAp stars as prime targets and eventuallly $\delta$ Scutis as secondary targets. I present here simulations of MOST observations of such targets with different rotation periods, in advance of the real data expected in the months after the scheduled 30 June 2003 launch.
\end{abstract}

$v \sin i$

All my troubles start

When you're too high.

Why did Slettebak ever try

To classify by

$v \sin i$ ?

$\mathrm{Y}, \mathrm{Z}, \mathrm{CNO}$

Abundance ratios have gone astray.

My models did something wrong.

Now I long for Maeder and Meynet.

[Lyrics from an unpublished work by McCartney, Lennon et al. found in lost files from the 1969 Stellar Rotation Symposium.] 


\section{Rotation: Blessing or curse?}

Rotation can make life complicated for an astronomer hoping to interpret stellar pulsations. It can distort the star's equipotential, as in Be and T Tauri stars, invalidating the assumption of spherical symmetry. It can introduce mixing currents, such as meridional circulation. The resulting changes in element distribution and mean molecular weight gradients in the upper layers of the star can change the acoustically reflective properties of the atmosphere. And there can be a competition between rotation and magnetism - Coriolis vs. Lorentz forces - which distort the pulsations of the star, as seen in magnetic chemically peculiar pulsators (roAp stars).

But these same complications can also serve as valuable diagnostics. Rotation splits the azimuthal $m$ degeneracy in the eigenspectra of nonradial pulsation modes, giving clues to the identities of the modes. Rotational broadening of the spectral lines of nonradial pulsators makes it possible to identify modes through spectroscopic Doppler Imaging, sensitive to degrees $\ell$ much higher than are observable photometrically.

This symposium on Stellar Rotation is certainly long overdue from the standpoint of pulsation. In 1969, the only region of pulsational instability in the HR Diagram which was both recognised and reasonably well understood was the classical instability strip containing Cepheids, RR Lyraes and $\delta$ Scuti stars. The pulsations of $\beta$ Cephei stars were known but their excitation mechanism remained a mystery until the new OPAL opacities were published in the 1990's. The periodic variability of HL Tauri had just been discovered by Landolt (1967) but it was not yet understood to be a pulsator - the first of the class of $\mathrm{ZZ}$ Ceti stars. The five-minute oscillations of the Sun were first reported in 1960 (Leighton et al. 1960) but it wasn't until 1970 that their true origin as acoustic pulsations was recognised (Ulrich 1970; Toomre 1970). While rotation was taken to be universal among stars, pulsation was still a fairly rare phenomenon in 1969 .

Today, the pulsational map reveals territories which were uncharted thirty years ago. Many of those territories will be explored in this volume by other authors ( $g$-modes in $\beta$ Cephei and Slowly Pulsating B stars by Connie Aerts; the $p$-mode solar oscillations by Joergen Christensen-Dalsgaard; and the $g$-modes of pulsating white dwarfs and PG 1159 stars by Steve Kawaler).

That leaves me little ground in instability space, so I will concentrate on a region near the upper Main Sequence, around spectral types late-A to early-F. Fortunately, this is very fertile ground, since there are few stars in this region which can be considered "normal" (i.e., non-pulsating, not chemically peculiar, no strong orgnaised magnetic fields). It is here that we find the $\delta$ Scuti and rapidly oscillating Ap (roAp) stars. In both cases, upcoming space missions like MOST, COROT and Eddington offer exciting opportunities to probe rotational effects in these pulsators.

\section{Delta Scuti stars: Still frustrating after all these years}

The pulsations of these A-F-type stars on or near the Main Sequence have been seen both photometrically and spectroscopically. The periods range from about 0.02 to 0.25 day (frequencies: $50 \geq \nu \geq 4 \mathrm{c} / \mathrm{d}$ ) with $V$ amplitudes as large as 0.1 
mag, but often as small as detection limits of a few millimag. The $\delta$ Scuti stars are non-magnetic (to the point that there is an almost total exclusion between magnetism and $\delta$-Sct-type pulsation) and have rotational characteristics typical of other non-peculiar A-F stars. Their projected rotational velocities cover a range of $50 \leq v \sin i \leq 150 \mathrm{~km} / \mathrm{s}$.

Multiperiodicity is common among $\delta$ Scutis, and the true richness of these stars' eigenspectra has only become apparent through coordinated multi-site photometric campaigns, such as the Delta Scuti Network (part of WET) and the STEPHI Network. However, as rich as the observed frequency spectra can be (29 frequencies in BI CMi (Breger et al. 2002). models of these stars indicate that many more frequencies can be potentially excited. It is a challenge (which has not yet been met) to match uniquely a set of observed frequencies to one of the many densely-populated theoretical eigenspectra covering even a small parameter space of mass and temperature.

One way to face the challenge of seismology of $\delta$ Scuti stars is to identify the pulsation modes $(\ell, m, n)$ associated with several or all of the observed frequencies, so they can be matched to eigenfrequencies of known modes in the stellar models. This is where rotation could come to the rescue.

\subsection{Doppler Imaging of modes}

Consider the spectral line profile of a rotating star, with inclination $i \sim 90^{\circ}$, observed with a spectrograph with sufficient resolving power $R=\lambda / \Delta \lambda$ such that $(v \sin i) / c>1 / R$.

A cooler (darker) spot at a point on the disk will produce a bump at the corresponding projected velocity in the line profile. Additional spots add more structure to the profile. Now extend this to a nonradial pulsation pattern of $(\ell, m)$. As the star rotates, the bumps travel across the profile from blue to red. If the pulsational wave has some velocity in the rest frame of the star, then the wave pattern progresses with some phase velocity which depends on the rotation rate, the wave speed, and whether the wave is pro- or retrograde. The number of bumps visible in the profile depends on the $\ell$ value.

An example of such travelling bumps in the residuals of the line profile is shown in Figure 1 for the $\delta$ Scuti star $\tau$.Pegasi from Kennelly et al. (1998). These data were obtained with the Advanced Fiber Optic Echelle (AFOE) spectrograph at the Fred Lawrence Whipple Observatory on Mount Hopkins, Arizona. This star has $v \sin i=150 \mathrm{~km} / \mathrm{s}$. A 2-D Fourier decomposition of these residuals reveals a rich mode spectrum with degrees up to $\ell=20$ which would be undetectable in integrated light photometry, and with frequencies below about $35 \mathrm{c} / \mathrm{d}$. Kennelly et al. (1998) conclude that the observed spectrum can be explained if the modes of $\tau$ Peg propagate in the prograde direction with $\ell \sim|m|$ and with frequencies that are about equal in the corotating frame of the star. This is just a hint of the diagnostic power possible through Doppler Imaging of rotating nonradial pulsators observed with sufficient spectral resolution.]

\subsection{Rotational splitting}

The handicap in $\delta$ Scuti star seismology is that these stars pulsate in radial and non-radial modes of low degree $\ell$ and low overtone $n$, and that many of them may exhibit mixed-mode behaviour, with both $p-$ and $g$-modes excited. Therefore, 


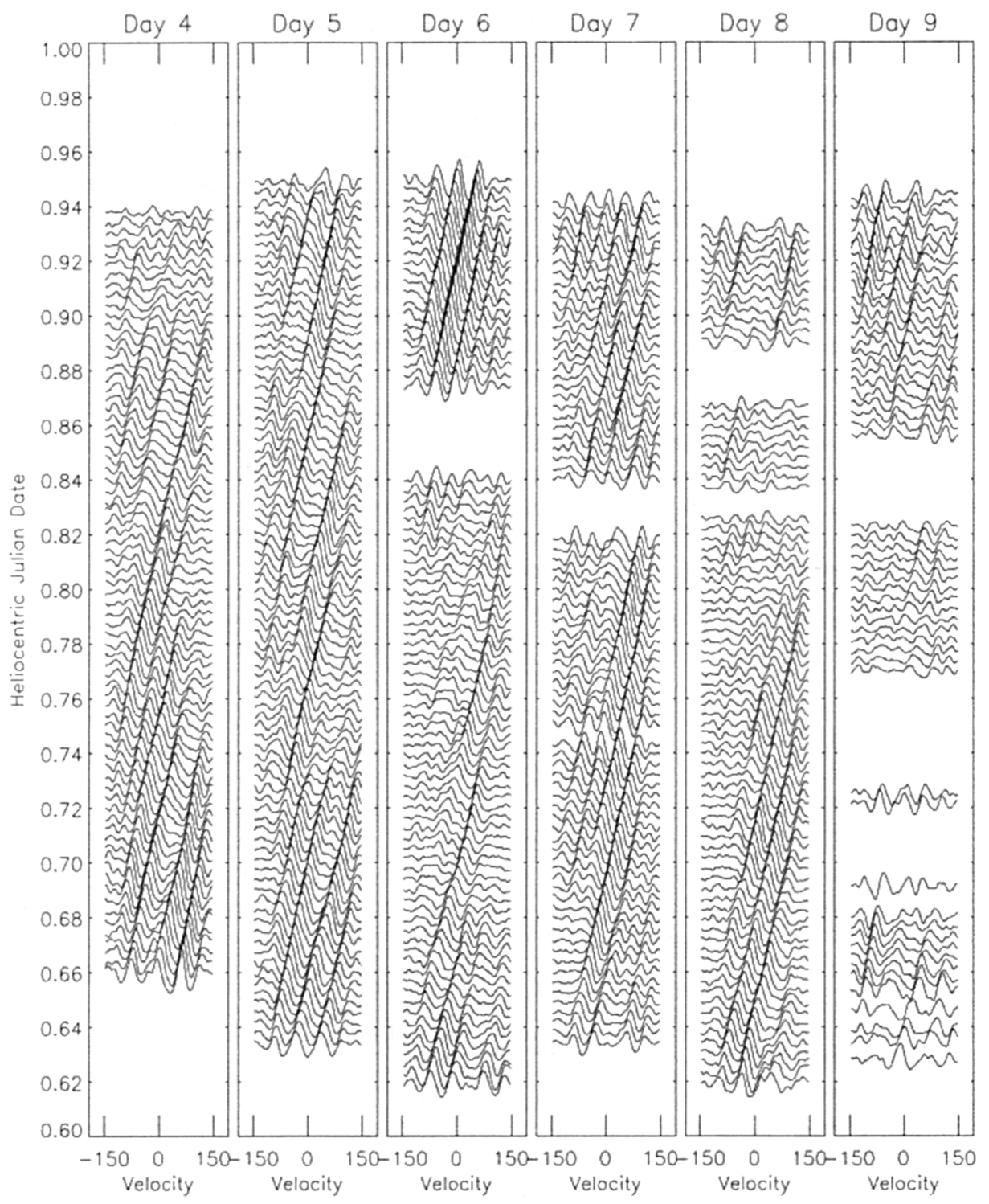

Figure 1. Spectral residuals of line profiles of the $\delta$ Scuti star $\tau$ Pegasi observed over several nights. The travelling bumps apparent in these residuals are due to nonradial pulsations whose phase veloicity across the visible disk of the star is a function of the wave velocity in the corotating frame and the rotation rate of the star. 
it is essential to identify a priori the pulsation modes $(\ell, m, n)$ associated with several or all of the observed frequencies, so they can be matched to the subsets of eigenfrequencies of known modes in the stellar models. One potential way to identify the degree $\ell$ of a mode in a star is to measure the multiplet of frequencies caused by rotation which splits the degeneracy among different values of $m=-\ell,(-\ell+1), \ldots 0, \ldots(\ell-1), \ell$.

\section{The MOST advantage}

MOST is a small (15-cm aperture) optical telescope and CCD photometry aboard a suitcase-sized microsatellite platform (mass $54 \mathrm{~kg}$, dimensions $60 \times 60 \times$ $30 \mathrm{~cm}$ ) - see Figure 2 - which is due to be launched from the Plesetsk Cosmodrome in northern Russia on 30 June 2003. MOST stands for "Microvariability \& Oscillations of STars", since it is designed to detect periodic variations in the brightnesses of nearby stars $(V<6)$ down to a precision of 1 micromag ( 1 part per million). Such a precision is about two orders of magnitude below current detection thresholds set by scintillation in the Earth's atmosphere. Existing space observatories like the Hubble Space Telescope do not have the photometrically stabilised instrumentation or an orbit with a large enough Continuous Viewing Zone (CVZ) to obtain data of this precision. So that's where the tiny MOST a.k.a. Canada's "Humble" Space Telescope - fills a unique and powerful niche. A more detailed description of the MOST experiment is provided by Walker, Matthews et al. (2003).

\subsection{The Instrument}

The MOST Instrument consists of four major subsystems (see Figure 3). (1) A flat periscope mirror directs light to (2) an optical telescope of only $15 \mathrm{~cm}$ in aperture, which feeds (3) a passively-cooled camera with twin CCDs serving as science detector and startracker, both governed by (4) low-power controller electronics.

The periscope mirror is necessary to fit the telescope sideways into the payload volume restrictions. The aperture of the telescope was also limited by volume and mass constraints, but a $15-\mathrm{cm}$ mirror gives good photon statistics for stars down to $V=6$. There was no room or money to install an independent startracking telescope for attitude control, so that function was incorporated into the instrument. This demanded a large field $\left(\sim 2^{\circ}\right)$ of the telescope, so a Maksutov design was adopted. Baffles reduce parasitic light in the system by a factor of $10^{-12}$. To keep the design simple, robust and inexpensive, there is only a single broadband optical filter $(350 \leq \lambda \leq 700 \mathrm{~nm})$. The blue cutoff matches the drop-off in quantum efficiency of the Marconi (now E2V) 47-20 CCDs used; the red cutoff is meant to avoid interference fringes at longer wavelengths in these thinned devices. The passive thermal control system - a cold-finger with trim heaters connected to a radiator looking out into cold space - will maintain the CCD operating temperature at around $-40 \pm 0.1 C$ with a minimum of power. The readout electronics allow the attitude control CCD to be read out at a rate of about $1 \mathrm{~Hz}$ with low $\mathrm{S} / \mathrm{N}$ while the science CCD can be read out as often as 5 times per minute with a $\mathrm{S} / \mathrm{N}$ as high as 220 per pixel for each stellar target. 


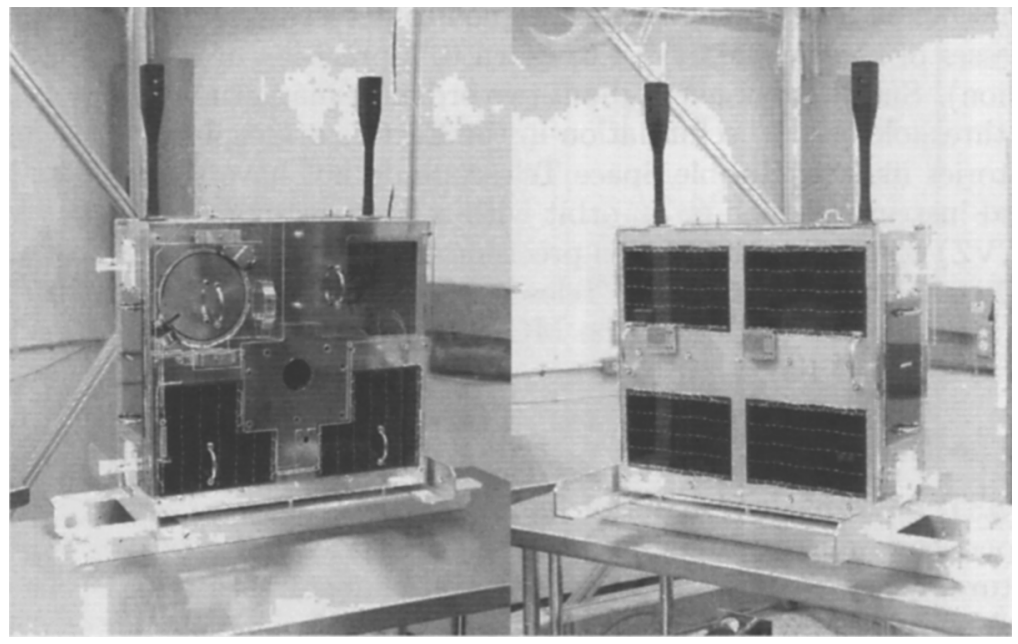

Figure 2. Two views of the MOST microsat. In the left image are visible the protective door covering the Instrument aperture, the location of the thermal radiator, and the rocket attachment area. On the right is shown the side covered with solar panels which will face the Sun during normal operations, as well as sun sensors for orientation. The two booms on top hold magnetometers which measure the local terrestrial magnetic field as an attitude control indicator. S-band antennae are mounted on the narrow sides. 


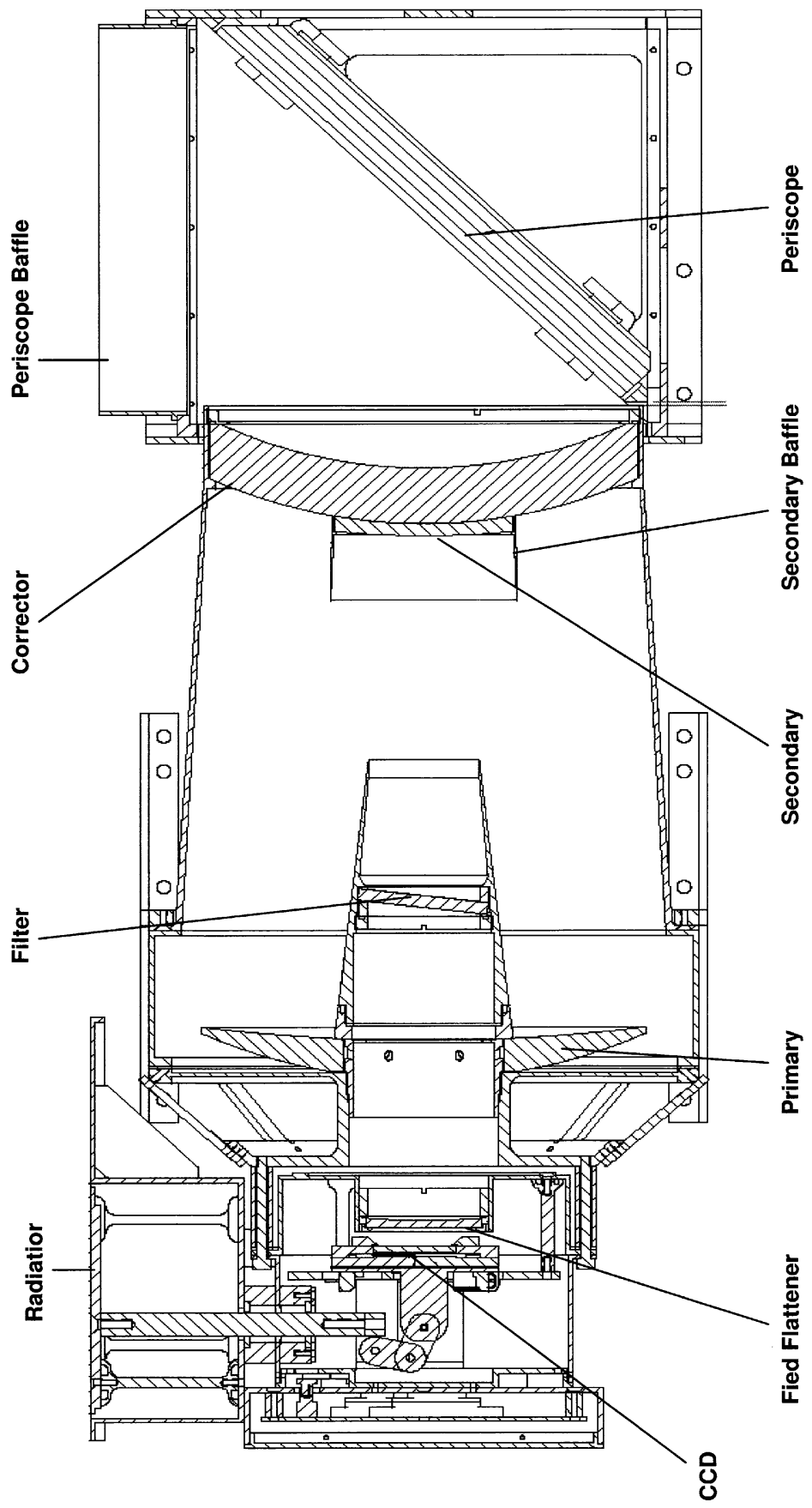

Figure 3. The layout of the MOST Instrument. 
Although the pointing accuracy of MOST is an improvement of two orders of magnitude over existing microsats with comparably small inertia, it is still relatively poor pointing for an astronomical instrument. Hence, the MOST photometer is equipped with an array of Fabry microlenses to project images of the entrance pupil of the telescope, illuminated by the target starlight, onto the science CCD. Since the telescope optics are not moving appreciably with respect to the camera focal plane, this image remains fixed on the detector even as the telescope pointing wanders. Unlike even a defocused moving image of the star, this extended Fabry image (covering about 1400 pixels of the CCD) makes the collected signal surprisingly immune to flatfield sensivity gradients, even at the sub-pixel scale. For stars as faint as $V=6$, a total $\mathrm{S} / \mathrm{N}$ of about 8300 can be achieved for exposures shorter than $60 \mathrm{sec}$.

\subsection{The Mission}

MOST is scheduled to be launched on 30 June 2003 into a low-altitude $(820 \mathrm{~km})$ circular polar orbit aboard a Russian "Rockot" (a former Soviet ICBM slated for destruction by arms limitation treaties) from the Plesetsk Cosmodrome.

It will be inserted at an inclination so the orbit will precess at the sidereal rate. This Sun-synchronous orbit will keep MOST above the terminator of the Earth. From this vantage point, the telescope will always look over the shadowed limb of the Earth, minimising stray Earthlight which could interfere with the ultraprecise photometry. This orbit also provides a Continuous Viewing Zone $(\mathrm{CVZ})$ in the sky spanning declinations $+34^{\circ} \leq \delta \leq-18^{\circ}$. Stars passing through the center of this band will remain visible continuously for up to 8 weeks.

The MOST primary science target list is limited to stars brighter than $V \sim 6$ which fall within the CVZ. However, this list features several Sun-like stars (including ones with known close-in exoplanet companions), metal-poor subdwarfs, roAp stars, and Wolf-Rayet stars. The Primary Science Target lists for Years 1 and 2 of the mission, as well as the list from which these targets were selected, can be found on the MOST UBC website: www.astro.ubc.ca/MOST

MOST was designed to achieve the mission's primary goal of detecting rapid photometric oscillations (periods of several minutes) in bright stars of these types, with precisions approaching 1 part per million $(1 \mu \mathrm{mag})$. Although MOST is a non-differential photometer, the relatively high frequencies of the periodic oscillations can be clearly distinguished in a Fourier spectrum of the data from lower-frequency modulations, drifts and noise. Such terms include orbital variations whose period $\left(P_{\text {orb }} \simeq 100 \mathrm{~min}\right.$ ) and harmonics will be accurately known after launch, and granulation noise in the stars themselves, which has a pronounced $1 / f$ frequency dependence based on the solar example.

\section{Rotational splitting as seen by MOST}

The MOST space-based photometer could potentially monitor bright $\delta$ Scuti stars in its Continuous Viewing Zone for up to 8 weeks, with an amplitude detection limit of only a few $\mu$ mag. However, the rotational splitting in these stars is not simple, so even the improved frequency resolution (approx. $0.2 \mu \mathrm{Hz}$ ) and sensitivity (1 ppm) possible from space do not necessarily yield unambiguous results, as can be seen in the simulation of MOST data shown in Figure 4. The 


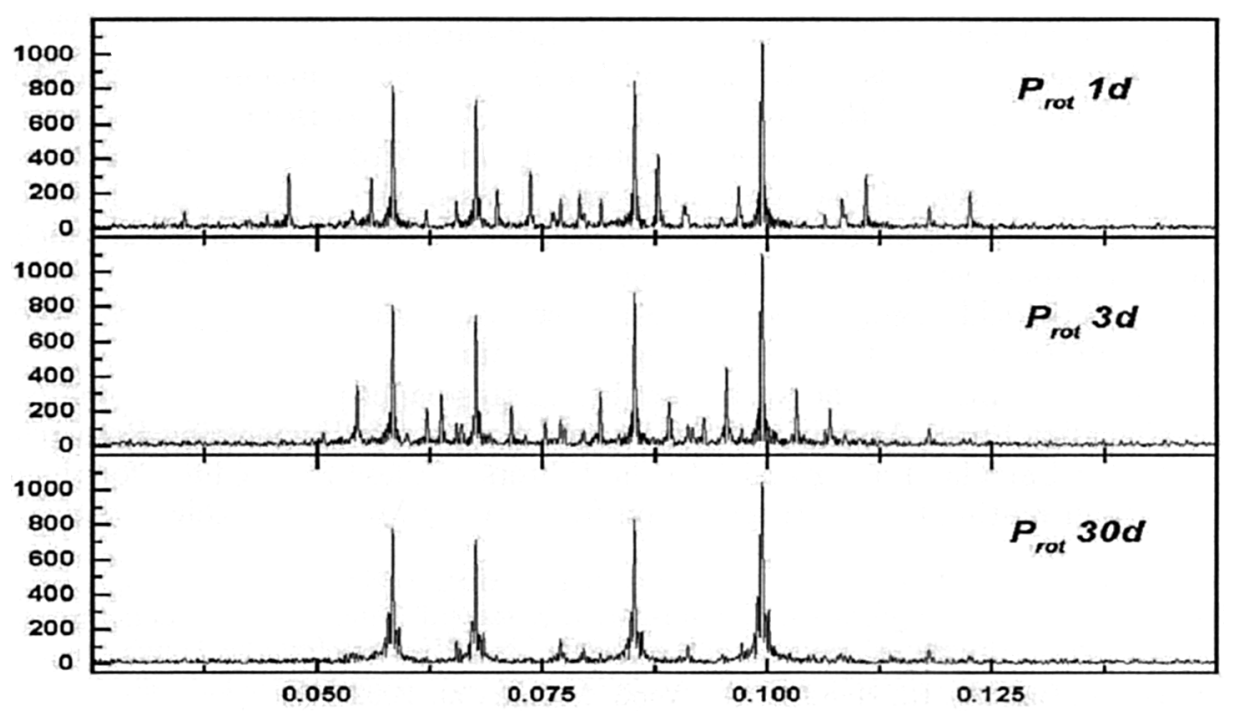

Figure 4. Fourier amplitude spectrum of a simulation of 50 days of MOST photometry of $\delta$ Scuti stars pulsating in modes of degree $\ell=2$ with three different rotation periods $P_{\text {rot }}$. Note the amplitude scale is in parts per million so the largest peak in this plot is only about 1 millimag.

stars simulated exhibit $\ell=2$ modes, with three different rotation periods. The resulting fine structure in the eigenspectrum is still complicated and adjacent modes can interfere with the interpretation of the rotational splitting.

Although $\delta$ Scuti stars have 'normal' atmospheres, there is greater promise in studying rotational effects among the chemically peculiar and strongly magnetic Ap stars. Despite their surface anomalies and magnetism, Ap stars are expected to be similar in overall structure to $\delta$ Scuti stars, which cohabit an overlapping region of the HR Diagram. The best explanation for the atmospheric peculiarities is chemical diffusion, regulated by the magnetic field geometry. There seems to be a near-total exculsion of $\delta$ Scuti-type (low overtone $n$ ) pulsation among Ap stars, but a cool subset of the class - known as roAp stars - shows acoustic modes of low degree but high overtone $n>>\ell$.

In this regime, the eigenfrequencies exhibit a more regular pattern which can be approximated by an asymptotic treatment (Tassoul 1990). To first order, the frequencies have a nearly equal spacing $\Delta \nu$, which is sensitive to the mean density of the star. There is also a fine splitting $\delta \nu_{02}$ which breaks up the regular spacing; this splitting depends on the sound speed gradient in the star's core, which is sensitive to the remaining hydrogen and hence, the main-sequence age. (For an example of this behaviour, see Kurtz, Matthews et al. (1989) and Kurtz et al. (2002).)

There is also splitting due to rotation, where the multiplet sidelobes are spaced from the central frequency by integer multiples of the rotation frequency $\Omega_{\text {rot }}$. This structure is evident in the simulations of roAp stars, in particular for a $\ell=2$ mode in roAp stars with the same trial rotation periods as shown in 
Figure 4 for $\delta$ Scuti stars. While the simulations are for modes of degree $\ell=2$, the sidelobe structure is easily distinguished from adjacent modes and easier to interpret in the case of the roAp stars.

In many respects, asteroseismic interpretation of roAp stars does not require foreknowledge of the exact mode values, as in the case of $\delta$ Scuti stars. However, the strong magnetic fields also perturb the frequencies at levels which can be comparable to the fine splitting $\delta \nu_{02}$. This is a potential diagnostic of the magnetic field strength and geometry (see, e.g., Cunha 2002) but it can also complicate the interpretation of the eigenspectrum.

Acknowledgements. The work described in this paper was largely funded by the Canadian Space Agency and the Natural Sciences \& Engineering Research Council of Canada. I am grateful to the Editors of these Proceedings for their patience in waiting for this contribution from a busy MOST Mission Scientist.

\section{References}

Breger, M., Garrido, R., Handler, G., Wood, M.A., et al. 2002,

MNRAS 329, 531

Cunha, M. 2002, MNRAS 333, 47

Kennelly, E.J., Brown, T.M., Kotak, R., Sigut, T.A.A. et al. 1998, ApJ 495, 440

Kurtz, D.W., Matthews. J.M. et al. 1989, MNRAS 240, 881

Kurtz, D.W., Kawaler, S.D., Riddle, R.L. et al. 2002, MNRAS 330, L57

Tassoul, M. 1990, ApJ 358, 313

Walker, G.A.H., Matthews, J.M., Kuschnig, R., Johnson, R., Rucinsky, S., Paxder. J., Burley, G., et al. 2003, PASP, in press 